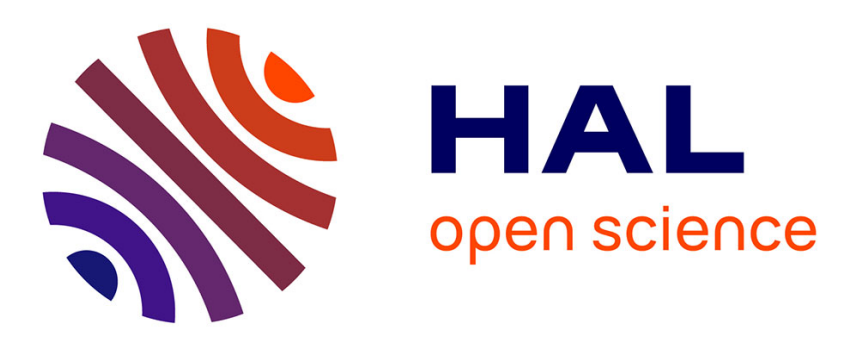

\title{
Muscle Fatigue Tracking Based on Stimulus Evoked EMG and Adaptive Torque Prediction
}

Qin Zhang, Mitsuhiro Hayashibe, David Guiraud

\section{To cite this version:}

Qin Zhang, Mitsuhiro Hayashibe, David Guiraud. Muscle Fatigue Tracking Based on Stimulus Evoked EMG and Adaptive Torque Prediction. ICRA: International Conference on Robotics and Automation, May 2011, Shanghai, China. pp.1433-1438. lirmm-00588948

\section{HAL Id: lirmm-00588948 https://hal-lirmm.ccsd.cnrs.fr/lirmm-00588948}

Submitted on 27 Apr 2011

HAL is a multi-disciplinary open access archive for the deposit and dissemination of scientific research documents, whether they are published or not. The documents may come from teaching and research institutions in France or abroad, or from public or private research centers.
L'archive ouverte pluridisciplinaire HAL, est destinée au dépôt et à la diffusion de documents scientifiques de niveau recherche, publiés ou non, émanant des établissements d'enseignement et de recherche français ou étrangers, des laboratoires publics ou privés. 


\title{
Muscle Fatigue Tracking based on Stimulus Evoked EMG and Adaptive Torque Prediction
}

\author{
Qin Zhang, Mitsuhiro Hayashibe, and David Guiraud
}

\begin{abstract}
Functional electrical stimulation (FES) is effective to restore movement in spinal cord injured (SCI) subjects. Unfortunately, muscle fatigue constrains the application of FES so that output torque feedback is interesting for fatigue compensation. Whereas, inadequacy of torque sensors is another challenge for FES control. Torque estimation is thereby essential in fatigue tracking task for practical FES employment. In this work, the Hammstein cascade with electromyography (EMG) as input is applied to model the myoelectrical mechanical behavior of the stimulated muscle. Kalman filter with forgetting factor is presented to estimate the muscle model and track fatigue. Fatigue inducing protocol was conducted on three SCI subjects through surface electrical stimulation. Assessment in simulation and with experimental data reveals that the muscle model properly fits the muscle behavior well. Moreover, the time-varying parameters tracking performance in simulation is efficient such that real time tracking is feasible with Kalman filter. The fatigue tracking with experimental data further demonstrates that the proposed method is suitable for fatigue tracking as well as adaptive torque prediction at different prediction horizons.
\end{abstract}

\section{INTRODUCTION}

Functional electrical stimulation (FES) is one of the existing solution to partly restore the lost motor function in persons with spinal cord injury (SCI). The electrical stimulus can artificially generate action potential on the axons of the alpha motor neurons to drive the muscle contraction instead of central nervous system. To date, FES has been used over a wide range of FES-aided support of standing, gait, grasp, or foot drop correction and tremor compensation.

In these applications, the stimulation is required to provide strong, consistent muscle force. It is well known, however, that muscles get fatigued more rapidly when artificially stimulated than when excited by the central nervous system due to the way which the motor units are recruited: inverse size principle, synchronized activation of motor units and constant order of recruitment. As a result, successful implementation of FES paradigms for rehabilitation has been greatly constrained by the early occurrence of fatigue. In addition, since SCI individuals have also lost sensory pathways, they cannot perceive their muscle fatigue as stimulation proceeds. This leads to movement failures and sub-optimal FES parameters tuning in practical applications.

Several techniques have been developed for muscle fatigue tracking. A fitness function was introduced to describe the effect of muscle fatigue on shank motion prediction induced

Q. Zhang, M. Hayashibe, and D. Guiraud are with DEMAR team, INRIA Sophia Antipolis, and LIRMM, UMR 5506 CNRS UM2, 161 Rue Ada, 34095 Montpellier Cedex 5, France. \{qin.zhang, hayashibe, guiraud\}@lirmm.fr by FES [1]. Muscle fatigue was modeled based on the metabolic profiles to represent force reduction and recovery in intermittent stimulation [2], [3]. A fatigue model, coupled with the force model that predicts the fatigue induced by different stimulation patterns on different days during isometric contractions was reported [4]. However, due to high nonlinearity and dynamic complexities, these muscle models are difficult to implement in application and it remains difficult to estimate the parameters.

In order to take into account the effect of muscle fatigue for FES control, reliable sensors are required to produce desired feedback or feed-forward signals. However, torque measurement through external sensors might be affected by slight voluntary motion, recording missing or other disturbances, and the devices, such as torque measuring chair, are not convenient at all for patients' daily use. The implanted sensor is a possible technique [5] but not available for practical use yet. As electromyography (EMG) signal was reported highly correlated with FES-induced torque in different muscle condition [6] [7], it was demonstrated able to be used as fatigue indicator. A predictive model using EMG instead of electrical stimulation as model input was developed in [8], and it was underlined evoked EMG could be used as a synthetic torque sensor. In [9], it was suggested to combine time and frequency domain variables for better prediction of force. Embedded in these works, a fixed relationship between EMG and force/torque during sustained stimulation was assumed. Nevertheless, some researchers found nonlinear relationship and dissociation between myoelectrical and mechanical activity in different fatigue states [10] or in the recovery process [11]. The evidence figures out the limitation of fatigue prediction strategies based on models with fixed parameters. Therefore, it is essential to find a suitable model in order to properly represent various muscle behavior.

The present work focuses on developing a method to predict the FES-induced mechanical contraction behavior during different muscle fatigue states. Online estimation and fatigue prediction are presented, based on a time-varying myoelectrical mechanical model, where the parameters are estimated by Kalman filter (KF) with forgetting factor. In terms of the improvements in fatigue tracking, the proposed method can be directly applied for fatigue prediction when perfect sensor data are available. Furthermore, if the measurements of the sensors are affected by external disturbances, the method can bridge the disturbances and provide sufficient fatigue tracking accuracy. 


\section{Myoelectrical Mechanical Model of Electrically Stimulated Muscle}

\section{A. Model Structure}

The discrete-time Hammerstein cascade is used to model electrically stimulated muscle, relating FES-evoked EMG to induced torque under isometric condition. This model structure has ever been used to represent some highly nonlinear systems, for example, biomechanical systems, such as stretch reflex [12] and electrically stimulated muscle, but relating stimulation to muscle force under isometric conditions [13]. It consists of a memoryless nonlinear part followed by a linear dynamic part as shown in Fig. 1.

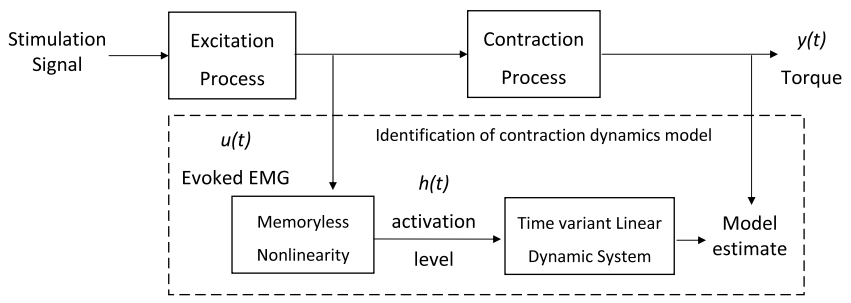

Fig. 1. Muscle model structure. The present work focus on the contraction dynamics model contained in the rectangle with dashed line. The memoryless nonlinearity models muscle activation level, $h(t)$, as a $n$ th-order polynomial function of the instantaneous EMG signal, $u(t)$. The linear dynamics is chosen as an ARX model.

In this work, the linear part is chosen as an autoregressive with external input (ARX) model, which has been shown experimentally to yield good prediction of output force/ torque in isometric situation [14]. Given model order $(l, m)$, it can be described in operator notation:

$$
A\left(z^{-1}\right) y(t)=B\left(z^{-1}\right) h(t)
$$

where $y(t)$ is the muscle torque at time instant $\mathrm{t}, h(t)$ is the activation level of muscle. $A(z)$ and $B(z)$ are the polynomials with respect to the backward shift operator $z^{-1}$, defined by the following equations:

$$
\begin{aligned}
& A\left(z^{-1}\right)=1+a_{1} z^{-1}+a_{2} z^{-2}+\cdots+a_{l} z^{-l} \\
& B\left(z^{-1}\right)=b_{1} z^{-1}+b_{2} z^{-2}+\cdots+b_{m} z^{-m}
\end{aligned}
$$

The memoryless nonlinear part maps the system input, i.e., instantaneous EMG signal $u(t)$, to $h(t)$. Traditionally, it is modeled with a $n$th order polynomial, in which case,

$$
h(t)=\sum_{i=1}^{n} \gamma_{i} u^{i}(t)
$$

Substituting (2) and (3) into (1) and expanding, the generated output at a given instant $t$ can be parameterized as:

$$
y(t, \mathbf{g})=\sum_{i=1}^{l} a_{i} y(t-i)+\sum_{i=1}^{m} \sum_{j=1}^{n} \mu_{i j}(u(t-i))^{j}
$$

where $\mu_{i j}=b_{i} \gamma_{j}, \mathbf{g}=\left[a_{1}, \cdots, a_{l}, \mu_{11}, \cdots \mu_{m n}\right]^{T}$ is a vector containing the muscle model coefficients. At a given instant $t$, the computation of $k$-step future output estimations using (4) is performed by assuming the system is stationary during the prediction horizon. The elements of $\mathbf{g}$ as well as the torque versus the EMG features are time-varying due to the effects of fatigue and the associated biochemistry. Moreover, model reidentification can effectively improve prediction performance in different muscle states [15], which inspires us to employ Kalman filter to estimate the timevarying model parameters online, and improve torque prediction for fatigue tracking.

\section{B. State-Space Representation of Time-Varying Model}

For the use of KF algorithm, a model representation in state space form is required. Such a model consists of process equation and measurement equation. Generally, for a model $(l, m, n)$, its state-space model can be written in a compact form:

1) process equation

$$
\mathbf{x}_{k}=\mathbf{f}\left(\mathbf{x}_{k-1}, u_{k-1}\right)=\mathbf{A} \mathbf{x}_{k-1}+\sum_{i=1}^{n} \mathbf{B}_{i}\left(u_{k-1}\right)^{i}
$$

2) measurement equation

$$
y_{k}=\mathbf{C x}_{k}
$$

In (5), $u$ is model input, $\mathbf{x}=\left[x_{1}, x_{2}, \cdots, x_{q}\right]^{T}$ is called state vector, $q=\max \{l, m\}$. The $q \times q$ matrix $\mathbf{A}$ relates the state at the previous time step $\mathbf{x}_{k-1}$, to the state at the current step $\mathbf{x}_{k}$. The $q \times 1$ matrix $\mathbf{B}_{i}$ relates the model input at the previous time step $\left(u_{k-1}\right)^{i}, i=1,2, \cdots, n$, to the state at the current step $\mathbf{x}_{k}$. They are represented as

$$
\mathbf{A}=\left[\begin{array}{cccccc}
a_{1} & 1 & 0 & \cdots & 0 & 0 \\
a_{2} & 0 & 1 & \cdots & 0 & 0 \\
\vdots & \vdots & \vdots & \ddots & \vdots & \vdots \\
a_{q-1} & 0 & 0 & \cdots & 0 & 1 \\
a_{q} & 0 & 0 & \cdots & 0 & 0
\end{array}\right], \quad \mathbf{B}_{i}=\left[\begin{array}{c}
\mu_{1, i} \\
\mu_{2, i} \\
\vdots \\
\mu_{q-1, i} \\
\mu_{q, i}
\end{array}\right]
$$

In practice, the matrices $\mathbf{A}$ and $\mathbf{B}_{i}$ might change with each time step due to time-varying property of muscle fatigue.

The $y$ in (6) is the measurement of output torque. The $1 \times q$ matrix $\mathbf{C}$ relates the state at the current step $\mathbf{x}_{k}$, to the measurement at the current step $y_{k}$ with the following expression:

$$
\begin{aligned}
& \mathbf{C}=\left[\begin{array}{lllll}
1 & 0 & \cdots & 0 & 0
\end{array}\right] \\
& \text { III. Identification OF The Myoelectrical } \\
& \text { Mechanical Muscle Model }
\end{aligned}
$$

In this study, a method to predict FES-induced torque only driven by EMG was developed, where KF with forgetting factor was employed to estimate the model states and parameters. The EMG and ankle torque will be used as model input and output for estimation.

\section{A. Kalman Filter with Forgetting Factor}

For online estimation and prediction, a KF was employed for the recursive estimation of the model parameters. The $\mathrm{KF}$ is an efficient recursive filter that estimates the internal states and parameters of a discrete-time system from a series of noisy measurements. It has some advantages in 
explicit modeling of the uncertainties associate with the proposed model and output measurements. Online estimation of the states in $\mathbf{x}$ and model parameters in $\mathbf{g}$ is investigated simultaneously. Parameter estimation with KF is performed considering the unknown parameters as elements of the state vector. In this way, the basic KF algorithm does not need to be changed except that the state vector $\mathrm{x}$ will be augmented with the unknown parameters. That is, the meta-state vector $\mathbf{w}$ has the expression $\mathbf{w}=\left[\begin{array}{ll}\mathbf{x}^{T} & \mathbf{g}^{T}\end{array}\right]^{T}$. The parameters in $\mathrm{g}$ are assumed time-invariant or slowly varying comparing to the process, that is, $\mathbf{g}_{k}=\mathbf{g}_{k-1}$. The augmented system is then described by

$$
\begin{aligned}
\mathbf{w}_{k} & =\mathbf{F}\left(\mathbf{w}_{k-1}, u_{k-1}\right)=\left[\begin{array}{c}
\mathbf{f}\left(\mathbf{x}_{k-1}, u_{k-1}\right) \\
\mathbf{g}_{k}
\end{array}\right], \\
y_{k} & =\mathbf{H} \mathbf{w}_{k}=\left[\begin{array}{ll}
\mathbf{C} & \mathbf{0}_{1 \times(l+m * n)}
\end{array}\right] \mathbf{w}_{k}
\end{aligned}
$$

The recursive estimation of the state-space model using KF consists of two phases, prediction and correction. The main equations are given as follows. More details can be found in [16].

1) prediction phase:

$$
\begin{aligned}
& \hat{\mathbf{w}}_{k}^{-}=\mathbf{F}\left(\hat{\mathbf{w}}_{k-1}, u_{k-1}\right) \\
& \mathbf{P}_{k}^{-}=\mathbf{D}_{k} \mathbf{P}_{k-1} \mathbf{D}_{k}^{T}+\mathbf{Q}_{k-1}
\end{aligned}
$$

2) correction phase:

$$
\begin{aligned}
\mathbf{K}_{k} & =\mathbf{P}_{k}^{-} \mathbf{H}_{k}^{T}\left(\mathbf{H}_{k} \mathbf{P}_{k}^{-} \mathbf{H}_{k}^{T}+\mathbf{R}_{k}\right)^{-1} \\
\hat{\mathbf{w}}_{k} & =\hat{\mathbf{w}}_{k}^{-}+\mathbf{K}_{k}\left(y_{k}-\mathbf{H} \hat{\mathbf{w}}_{k}^{-}\right) \\
\mathbf{P}_{k} & =\left(\mathbf{I}-\mathbf{K}_{k} \mathbf{H}_{k}\right) \mathbf{P}_{k}^{-}
\end{aligned}
$$

Equations (9), (10) project the state and error covariance estimates forward from time step $k-1$ to step $k$. The $\hat{\mathbf{w}}_{k-1}$ and $\mathbf{P}_{k-1}$ are initial estimates for the state and for the measurement variance, respectively. $\mathbf{R}_{k}$ is measurement noise covariance, while $\mathbf{Q}_{k}$ is process noise covariance. $\mathbf{D}_{k}$ is the Jacobian matrix of the plant transfer functions with respect to the involved variables at step $k$, with each element $D_{[i, j]}$ computed as:

$$
\mathrm{D}_{[i, j]}=\frac{\partial \mathrm{F}_{[i]}}{\partial \mathrm{w}_{[j]}}\left(\hat{\mathbf{w}}_{k-1}, u_{k-1}\right)
$$

In correction phase, equations (11) - (13) adjust the projected estimates by an actual measurement at step $k . \mathbf{K}_{k}$ is called Kalman gain.

Although KF is an effective way to estimate the state and parameters of a discrete-time controlled process. Its performance in estimating the time-varying parameters is degraded since it refers to the entire history of the past measurements [17]. This is particularly troublesome since the myoelectrical and mechanical activity of stimulated muscle may vary with different fatigue states in prolonged or repetitive stimulation. Deliberately, forgetting factor $\lambda$ is incorporated to allow tracking of time-varying parameters [17]. Thus, equation (10) and (11) can be rewritten as

$$
\begin{aligned}
\mathbf{P}_{k}^{-} & =\mathbf{D}_{k} \mathbf{P}_{k-1} \mathbf{D}_{k}^{T} / \lambda \\
\mathbf{K}_{k} & =\mathbf{P}_{k}^{-} \mathbf{H}_{k}^{T}\left(\mathbf{H}_{k} \mathbf{P}_{k}^{-} \mathbf{H}_{k}^{T}+\lambda\right)^{-1}
\end{aligned}
$$

Choosing $\lambda \in[0,1]$ is based on how much we expect the filter to forget the past measurements. In most application, $\lambda$ is only slightly lower than 1 (for example, $0.85 \sim 1$ ). In our study, $\lambda$ is chosen to be 0.97 in simulation and 0.997 in experimental validation. These values represent a compromise between the smoothness of tracking and lag in detecting the changes in model parameters or muscle condition.

\section{B. Experimental Setup for Model Identification}

In order to investigate the estimation and prediction method proposed in this paper, experiments were conducted on SCI subjects in PROPARA rehabilitation center, Montpellier, France. The experimental setup, stimulation and recording electrodes configuration are depicted in Fig. 2. The subjects were seated on the chair with their ankle at
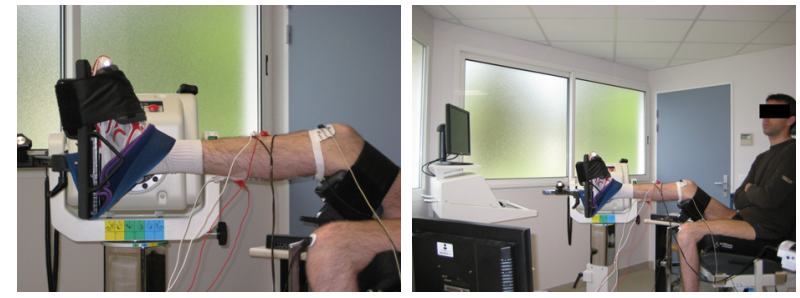

Fig. 2. Electrodes configuration (left) and experimental set-up (right).

$90^{\circ}$, while the foot was strapped on the pedal. Amplitude modulated electrical current pulses were sent to the the right triceps surae muscle via surface electrodes $(10 \mathrm{~cm} \times 3 \mathrm{~cm})$ and caused muscle contractions and ankle-joint motion in isometric condition. One electrode was placed $5 \mathrm{~cm}$ beneath the popliteal cavity and the other beneath the insertion point of the medial and lateral gastrocnemius on the Achilles tendon. The muscle group was stimulated with amplitude modulation at a constant frequency $(30 \mathrm{~Hz})$ and constant pulse-width $(450 \mu \mathrm{s})$, under isometric conditions by a portable stimulator (Cefar Physio 4, Cefar Medical AB, Lund, Sweden).

Evoked EMG activity of soleus were recorded, amplified (gain 1000) and sampled at $4 \mathrm{KHz}$ by an acquisition system (Biopac MP100, Biopac Systems Inc., Santa Barbara, CA, USA). Two bipolar AgCl EMG electrodes were positioned over muscle belly along the muscle fiber direction with $20 \mathrm{~mm}$ interelectrode spacing. The reference electrode was placed on the patella of another leg. The skin under the electrodes was shaved to minimize the impedance. Isometric ankle plantar-flexion torque was measured using a calibrated dynamometer (Biodex, Biodex Medical Systems, Inc., New York, USA), interfaced with the acquisition system (Biopac MP100) and sampled at $4 \mathrm{KHz}$.

The detailed experimental protocol has been shown previously [15]. The data used for this work include intermittent four series of five stimulation trials which were enforced to 
induce muscle fatigue. Each trial consists of 1s ramp, $2 \mathrm{~s}$ plateau at intensity evoking $50 \%$ of maximum torque, $1 \mathrm{~s}$ falling and $2 \mathrm{~s}$ interval between adjacent trials. It means each trial began and ended without other stimulations. Hence, if desired, data from successive trials could be concatenated for model estimation.

This study was approved by the ethical committee of France. Three SCI subjects participated in the experiments and signed informed consent form.

\section{IDENTIFICATION RESUlts}

The proposed model structure and KF estimation method were evaluated in simulations and with experimental data.

For a model $(l, m, n)$, we need to estimate $\max (l, m)+$ $(l+m * n)$ dimensional meta-state. As the stimulus amplitude is quite small at the beginning of stimulation, when initializing the filter, we choose the meta-state vector $\hat{\mathbf{w}}_{0}=0$ and the output estimate $\hat{y}_{0}=0$. The initial error covariance is $\mathbf{P}_{0}=\mathbf{I}$, where $\mathbf{I}$ is an identity matrix.

\section{A. Time-Varying Parameters Tracking in Simulation}

In simulation, invariant parameters tracking was evaluated with KF firstly to investigate the stability of the muscle model estimation. Secondly, since the muscle behavior represents gradual time-varying property with muscle fatigue, we slowly change the model parameters at different time instants to simulate the changes of muscle condition. The advantage of simulation is that the true parameters are known to be compared with the estimated ones. The drawback is that it is difficult to design suitable time-varying parameters in a high order model which will not cause too much variation in output. Thereby, we chose simulation model order $(2,2,1)$ to reduce the model complexity and have fewer parameters.

Fig. 3 shows the plant simulator output as well as the corresponding simulator input. The output estimate of KF is also shown in this plot. In the upper graph, the solid line indicates the actual model output, while the dotted line indicates its estimate. The corresponding parameter estimates of the model are depicted in Fig. 4. The solid lines indicate the true parameters, while the dotted lines indicate parameters estimates. All the parameters converge steadily after $5 \mathrm{~s}$ when the parameters are static. From $35 \mathrm{~s}, 50 \mathrm{~s}$ and $60 \mathrm{~s}$, the model parameters were changed gradually, the estimates track the changes well. Even at $35 \mathrm{~s}, a_{1}$ suddenly decreases, the estimate tracks the variance fast. The simulation results imply that the identification method is suitable for timevarying parameters tracking in muscle contraction model.

\section{B. Fatigue Tracking Based on Experimental Data}

The proposed estimation method was further evaluated with the experimental data on three SCI subjects. The data from successive trials were concatenated for estimation. The normalized MAV and normalized torque served as input and output, respectively. The signal was processed in four steps before estimation:

- Blanking window method is used to remove stimulation artifact from EMG raw signal and extract muscle response signal (Mwave);
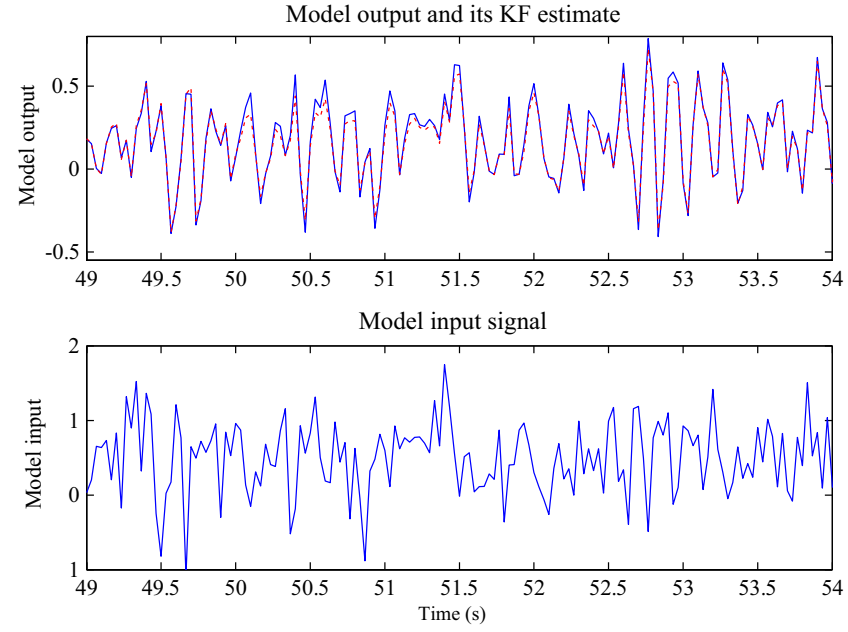

Fig. 3. Data set for model identification. Model input is chosen as random, Gaussian signal.

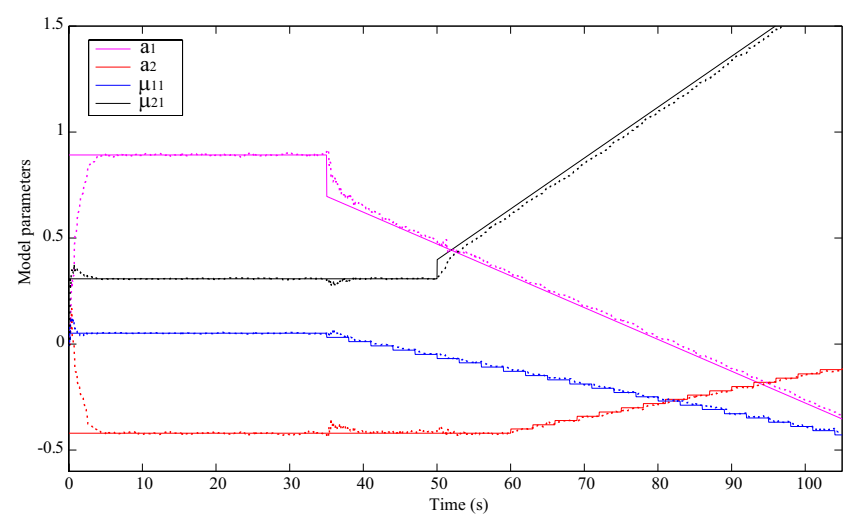

Fig. 4. Convergence and tracking of model parameter estimates for plant simulator identification. Solid lines indicate true parameters. Dotted lines indicate parameter estimates. All the initial estimates are set at zero.

- Lowpass filter of measured ankle torque (6th-order, cutoff frequency $100 \mathrm{~Hz}$ ) and measured EMG (6th-order, cutoff frequency $300 \mathrm{~Hz}$ );

- The filtered EMG signal is divided into epochs with each epoch containing one Mwave, the MAV is calculated every five epochs, the average torque is calculated in the same time window;

- The MAV and torque are normalized with respect to their maximum value.

During 100s intermittent stimulation, the torque of each subject decreased to different levels due to muscle fatigue. The nonlinear relationship between MAV of EMG and torque was revealed in experiments [15].

The selection of model order $(l, m, n)$ is a key stage of model parameters estimation. We chose $n=3$ for the polynomial function as proposed in [13]. The model order of linear part is determined by comparing the Rissanen's minimum description length (MDL) [18], which provides a criterion for tradeoff between model simplicity and model's fitness for the data. Finally model order $l=3, m=4$ was chosen with relatively less MDL value and simpler model. 
TABLE I

PREDICTION FILTER PERFORMANCE ON EXPERIMENTAL DATA

\begin{tabular}{c|c|c|c|c}
\hline \multirow{2}{*}{ Subject } & \multirow{2}{*}{ Averaged error } & \multicolumn{3}{|c}{ Prediction horizon } \\
\cline { 3 - 5 } & & $6 \mathrm{~s}$ & $18 \mathrm{~s}$ & $30 \mathrm{~s}$ \\
\hline \multirow{2}{*}{ S1 } & RMS & 0.0278 & 0.0314 & 0.0366 \\
\cline { 2 - 5 } & Peak & 0.0743 & 0.0962 & 0.1146 \\
\hline \multirow{2}{*}{ S2 } & RMS & 0.0524 & 0.0534 & 0.0556 \\
\cline { 2 - 5 } & Peak & 0.1208 & 0.1523 & 0.1697 \\
\hline \multirow{2}{*}{ S3 } & RMS & 0.0387 & 0.0418 & 0.0437 \\
\cline { 2 - 5 } & Peak & 0.1036 & 0.1360 & 0.1510 \\
\hline
\end{tabular}

1) Torque Prediction Performance: To investigate the torque prediction performance in time, different prediction horizons, 6s, 18s and 30s, are tested. The idea is illustrated in Fig. 5, featuring the estimated model at time instant $t 0$. The torque predictions are computed using (4), considering a static system within the prediction horizon. The root mean square (RMS) error and peak prediction error at every torque sample for a given prediction horizon are computed and then averaged. The prediction errors are quantified in Table I.

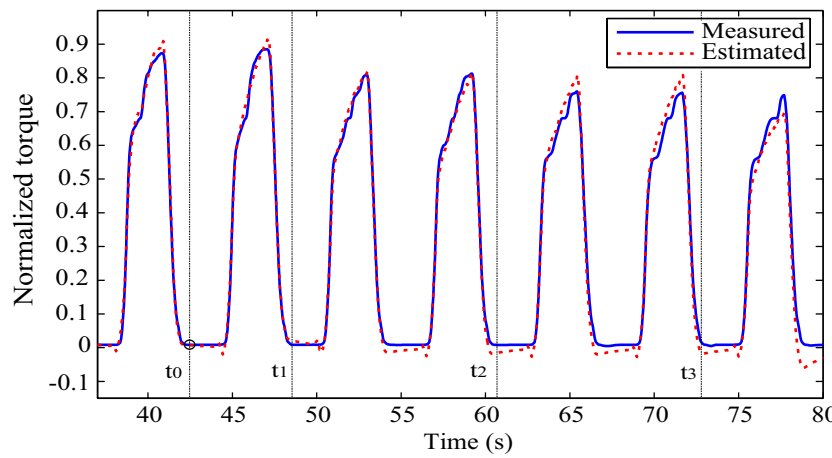

Fig. 5. Measured and estimated torque at time instant $t 0$. For evaluating prediction performance, the prediction errors for prediction horizon $t 1, t 2$, and $t 3(6 \mathrm{~s}, 18 \mathrm{~s}$ and $30 \mathrm{~s}$ respectively) are computed.

2) Predictive Performance with Fatigue Tacking: In order to evaluate the tracking robustness against recording missing, slight voluntary motion or failures, torque prediction is integrated to the fatigue tracking task. The event, such as torque recording error or disturbances, is assumed to result in tracking failures. The estimated model at $t 0$ and EMG signal are used to predict generated torque.

Fig. 6 reveals the prediction performance in subject1 when muscle torque decreases to $71 \%$ by the maximum torque during 100s-stimulation. Assuming the measurement is missing or interrupted from time $t 0$ to $t 1$, the estimated model at $t 0$ was used to predict the torque until instant $t 1$. When prediction is executed, the torque estimation is only driven by EMG signal, while the online model identification is suspended. To evaluate the prediction performance in different muscle fatigue states, this process is repeated until the end of stimulation, where every $18 \mathrm{~s}$ the measurement is suspended. We can find the prediction fits the measured torque well, suggesting that even if the torque measurement is interrupted, the predicted torque based on EMG can be used to bridge such gap for long-time application of FES.

The estimated parameters are partly depicted in Fig. 7. This plot shows the parameters denoting the weights of the past torque. It is obvious that the parameters vary gradually representing different myoelectrical mechanical correlation during fatigue. Whereas, the proposed muscle model and KF algorithm with forgetting factor provide a good prediction performance as shown in Fig. 6.

\section{Discussion for Fatigue Tracking Control}

The prediction result presented in Fig. 5 reveals good performance of the predictive filter, successively acquiring the muscle mechanical behavior during FES. In addition, the error values given in Table I indicate that the RMS error and peak error do not increase significantly when the prediction horizon is extended. It indicates the proposed myoelectrical mechanical muscle model fits properly the muscle behavior. If the prediction quality degrades when an expanded prediction horizon is selected, it is probably due to the difference of muscle fatigue levels. This can occur when prolonged or repetitive stimulation is delivered to the muscle. In this application, 18s prediction horizon, is considered as the optimal choice.

The myoelectrical mechanical muscle model has been described in previous work [8], with recursive least squares method for identification. However, there was no significant consideration for different fatigue condition in intermittent stimulation as illustrated in Fig. 6. Moreover, the proposed approach in this paper can be further expanded to use other measurement, like joint angle, or multiple measurements update with KF thus avoiding torque sensor.

In this paper, the proposed method represents feasible and effective torque prediction only based on EMG in isometric condition for fatigue tracking. It is also promising to be applied in dynamic condition by multiplying a force-length or force-velocity function to the proposed myoelectrical mechanical muscle model as proposed in [19].

\section{CONCLUSIONS AND FUTURE WORKS}

In this work, we propose a method to predict the FESinduced ankle torque based on a time-varying myoelectrical mechanical muscle model, where the model parameters are estimated by Kalman filter with forgetting factor. The proposed prediction method is applied in fatigue tracking task with satisfactory results. The future work will be extended to adaptive FES control for muscle fatigue compensation in SCI patients. It is also possible to introduce joint dynamics model and joint angle sensors along with the proposed method, then we will work on FES control with torque prediction based on EMG without the use of torque sensor.

\section{ACKNOWLEDGMENTS}

The first author is supported in part by China Scholarship Council (CSC). We would also like to warmly thank patients for their cooperation, Maria Papaiordanidou and Dr. Charles Fattal for their help to do the experiments. 
Measured torque (blue solid) and estimated torque (red dotted)

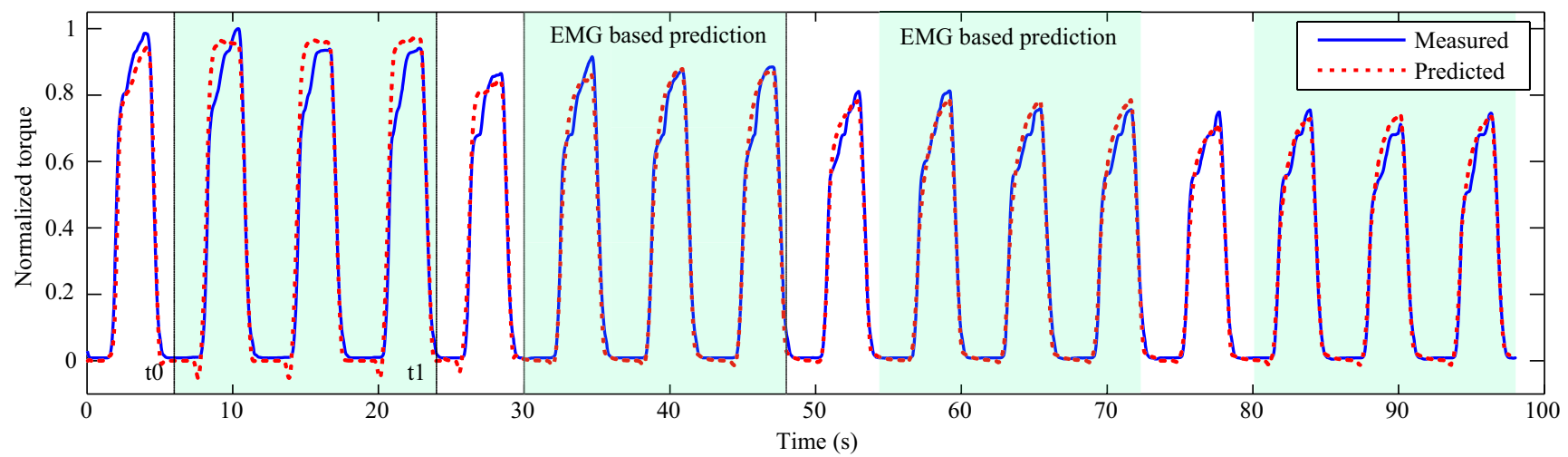

Fig. 6. Fatigue tracking based on EMG-torque model and Kalman filter in subject1. The model estimated at instant $t 0$ was used to predict the torque from instant $t 0$ to $t 1$, while the online identification and state estimation are switched off. This process is repeated until the end of stimulation. The blue solid line indicates the measured torque, while the red dotted lines indicate estimated or predicted torque (in the range with green background color).

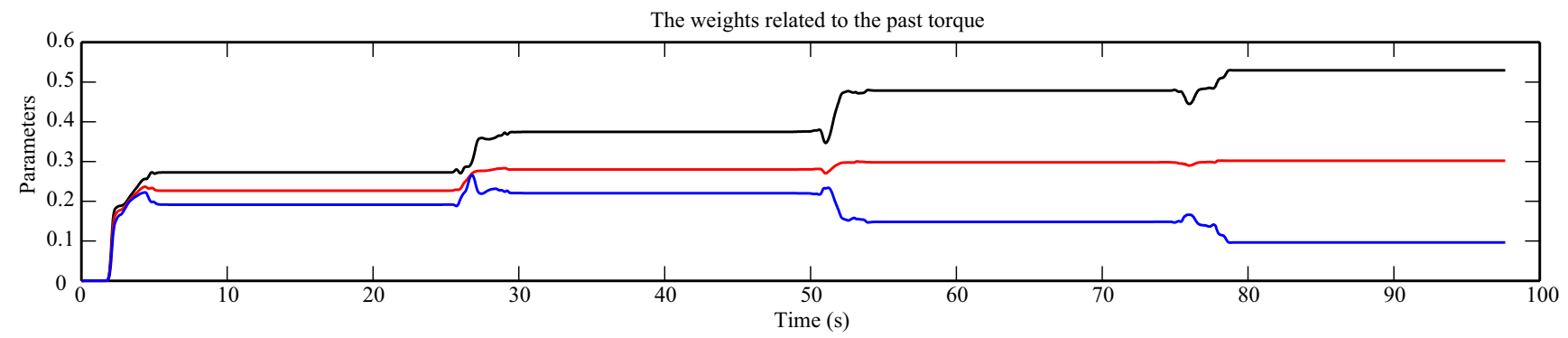

Fig. 7. Model parameters represent time-varying feature during different muscle states. Black, red and blue lines respectively indicate the weights of past torque $a_{1}, a_{2}$ and $a_{3}$ in equation (4).

\section{REFERENCES}

[1] R. Riener, J. Quintern and G. Schmidt, Biomechanical Model of the Human Knee Evaluated by Neuromuscular Stimulation, Journal of Biomechanics, vol. 29, 1996, pp. 1157 - 1167.

[2] J. Mizrahi, D. Seelenfreund, et al., Predicted and Measured Muscle Forces After Recoveries of Differing Durations Following Fatigue in Functional Electrical Stimulation, Artificial Organs, vol. 21, 1997, pp. 236-239.

[3] J. K. Lim, M. H. Nam and G. Khang, "Model of Activation Dynamics for an FES-Induced Muscle Fatigue", in 22nd Annual International Conference of the IEEE Engineering in Medicine and Biology Society, Chicago, IL, USA, vol. 3, 2000, pp. 2251-2253.

[4] J. Ding, A. S. Wexler, and S. A. Binder-Macleod, A predictive model of fatigue in human skeletal muscles, Journal of Applied Physiology, vol. 89, 2000, pp. 1322-1332.

[5] J. A. Hoffer, Closed-Loop, Implanted-Sensor, Functional Electrical Stimulation System for Partial Restoration of Motor Functions, United Stated Patent, vol. 19, 1988.

[6] N. C. Chesler and W. K. Durfee, Suface EMG as a Fatigue Indicator During FES-induced Isometric Muscle Contractions, Journal of Electromyography and Kinesiology, vol. 7, 1997, pp. 27-37.

[7] A. Erfanian, H. J. Chizeck, and R. M. Hashemi, "ExcitationContraction Fatigue During Sustained Electrical Stimulation of Paralyzed Muscle", in 18th Annual International Conference of the IEEE Engineering in Medicine and Biology Society, Amsterdam, Netherlands, 1996, pp. 1460-1461.

[8] A. Erfanian, H. J. Chizeck, and R. M. Hashemi, Using Evoked EMG as a Synthetic Force Sensor of Isometric Electrically Stimulated Muscle, IEEE Transaction on Biomedical Engineering, vol. 45, 1998, pp. 188202.

[9] D. Tepavac and L. Schwirtlich, Detection and Prediction of FESInduced Fatigue, Journal of Electromyography and Kinesiology, vol. 7, 1997, pp. 39-50.
[10] J. Mizrahi, E. Isakov and Z. Suzak, Myoelectric and Force Characteristics in Transcutanteous Isometric FES, Basic and Applied Myology, vol. 4, 1994, pp. 147-154.

[11] N. Y. Yu and S. H. Chang, "Mechanical and Electromyographic Response to Stimulated Contractions in Paralyzed Tibialis Anterior Post Fatiguing Stimulations", in 13th International Conference on Biomedical Engineering, Singapore, 2009, pp. 1667-1671.

[12] E. J. Dempsey and D. T. Westwick, Identification of Hammerstein Models With Cubic Spline Nonlinearities, IEEE Transaction on Biomedical Engineering, vol. 51, 2004, pp. 237-245.

[13] T. L. Chia, P. Chow and H. J. Chizeck, Recursive Parameter Identificaiton of Constrained Systems: An Applicaiton to Electrically Stimulated Muscle, IEEE Transaction on Biomedical Engineering, vol. 38, 1991, pp. 429-442.

[14] L. A. Bernotas, P. E. Crago, and H.J. Chizeck, A Discrete-Time Model of Electrically Stimulated Muscle, IEEE Transaction on Biomedical Engineering, vol. BME-33, 1986, pp. 829-838.

[15] Q. Zhang, M. Hayashibe, et al., "Torque Prediction Using Stimulus Evoked EMG and its Identification for Different Muscle Fatigue States in SCI Subjects", in 32nd Annual International Conference of the IEEE Engineering in Medicine and Biology Society (EMBC' 10), Buenos Aires, Argentina, 2010, pp. 3523-3526.

[16] G. Welch and G. Bishop, "An introduction to the kalman filter", Technical Report 95-041, University of North Carolina, Department of Computer Science, 1995.

[17] G. A. Mack and V. K. Jain, Speech Parameter Estimation by Timeweighted-Error Kalman Filter, IEEE Transaction on Acoustics, Speech and Signal Processing, vol. ASSP-31, 1983, pp. 1300-1303.

[18] J. Rissanen, "A Universal Prior for Integers and Estimation by Minimum Description Length", The Annals of Statistics, vol. 11, pp. 416 C 431, 1983.

[19] W. Farahat and H. Herr, "A Method Identification of Electrically Stimulated Muscle", in Proceeding of the 2005 IEEE Engineering in Medicine and Biology 27 th Annual Conference, Shanghai, China, 2005, pp. 6225-6228. 